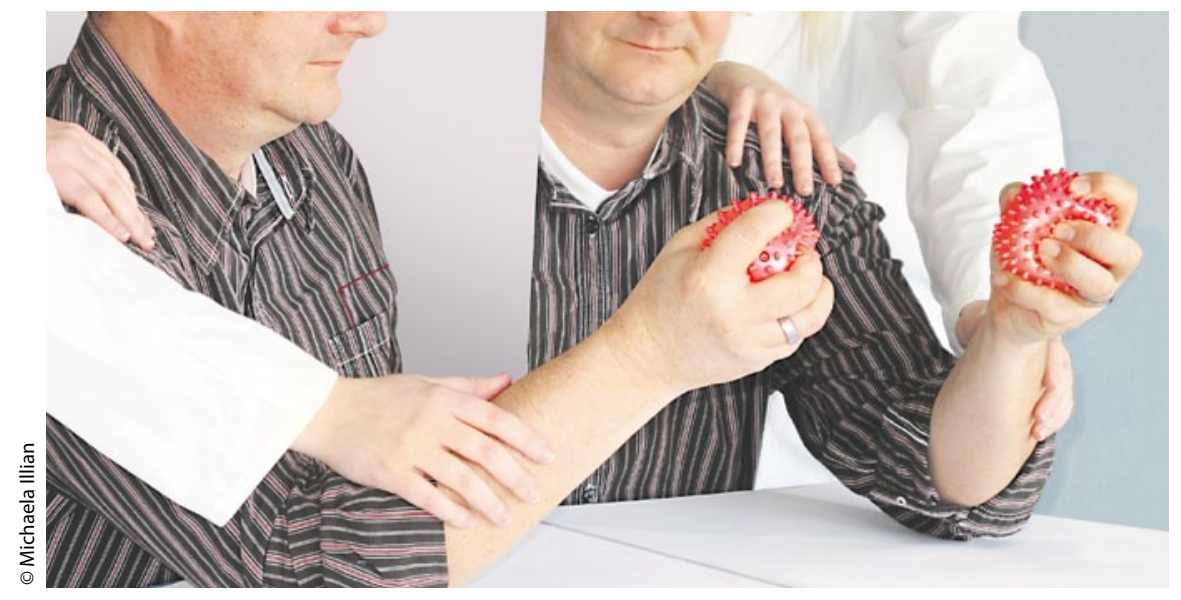

Hat die Spiegeltherapie nach Schlaganfall einen spezifischen Effekt oder nicht? Eine Cochrane-Analyse versuchte, das herauszufinden.

\section{Wie gut und auf was wirkt die Spiegeltherapie nach Schlaganfall wirklich?}

In dieser Cochrane-Analyse wurde der Schmerz nach einem Schlaganfall untersucht, das heißt, die Wirksamkeit eines Verfahrens auf verschiedene Folgezustände nach Schlaganfall. Die Wissenschaftler untersuchten 14 Studien, die die Spiegeltherapie mit anderen Interventionen nach einem Insult verglichen.

D ie Spiegeltherapie wird zunehmend populärer, es sind zahlreiche Studien hierzu erschienen. Ob es einen spezifischen Effekt dieser Therapie gibt, ist bislang aber immer noch nicht hinreichend geklärt. Die
Analyse identifizierte insgesamt 14 Studien, die die Spiegeltherapie mit anderen Interventionen nach einem Schlaganfall verglichen. Insgesamt zeigte die Spiegeltherapie einen signifikanten Effekt auf moto- rische Funktionen. Der Effekt auf Aktivitäten des täglichen Lebens war geringer, aber auch signifikant. Schließlich wurde auch ein signifikanter positiver Effekt auf den zentralen Deafferenzierungsschmerz nach Schlaganfall gefunden.

Trotz Limitierung (z. B. insgesamt nur 567 Teilnehmer in 14 Studien) konnte ein Effekt der Spiegeltherapie gezeigt werden, vor allem natürlich auf die motorischen Funktionen nach einem Insult, aber eben auch auf den Schmerz.

Kommentar: Diese Analyse belegt, dass die Spiegeltherapie auch in der Schmerztherapie ihre Berechtigung hat. Es ist bemerkenswert, dass der Einfluss dieser Therapie auf Deafferenzierungsschmerzen ähnlich groß ist wie auf motorische Funktionen. Dies ist jedoch laut Studien mit funktioneller Kernspintomografie zur Plastizität des Gehirns pathophysiologisch gar nicht unplausibel. Es wäre zu begrüßen, wenn die Spiegeltherapie noch spezifischer in der Schmerztherapie untersucht wird. In der rehabilitativen Schmerztherapie kann sie nach dieser Studie mit großer Berechtigung eingesetzt werden.

Prof. Dr. med. Stefan Evers

Thieme H, Mehrholz J, Pohl M, Behrens J, Dohle C. Mirror therapy for improving motor function after stroke. Cochrane Database Syst Rev 2012; 3: CD008449

\title{
Trigeminusneuralgiebehandlung mit Botulinumtoxin A
}

In der hier vorgelegten kleine Studie wurde Botulinumtoxin Typ A in der Behandlung der Trigeminusneuralgie untersucht. Dies ist insofern bemerkenswert, als für die Trigeminusneuralgie normalerweise gut wirksame und verträgliche Medikamente zur Verfügung stehen. Die Ergebnisse der Studie sind überzeugend und sollten jetzt im größeren Stil repliziert werden.

$\mathrm{n}$ diese Studie wurden 42 Patienten mit einer klassischen Trigeminusneuralgie eingeschlossen und entweder mit 75 Einheiten Botulinumtoxin A oder mit Kochsalzlösung behandelt. Das Injektionsgebiet entsprach dem Schmerzgebiet. Zusammenfassend reduzierte Botulinumtoxin A die Schmerzintensität nach zwei Wochen und die Attackenfrequenz nach einer Woche. Dieser Effekt wurde über die gesamten drei Monate der Studienperiode aufrechterhal- ten. Daher kann gefolgert werden: Botulinumtoxin $\mathrm{A}$ ist eine effektive und sichere Behandlung in der Trigeminusneuralgie.

Kommentar: Inzwischen ist nun auch die Trigeminusneuralgie als weitere Schmerzerkrankung in den Blickpunkt der Anwendung von Botulinumtoxin A gelangt. Diese Studie wurde methodisch weitgehend sauber durchgeführt, allerdings ohne Angabe, wie die Patienten zusätzlich zu Botulinumtoxin
A noch behandelt wurden. Zudem ist das Kollektiv nicht hinreichend klassifiziert. So ist nicht ganz deutlich, ob wirklich immer eine klassische Trigeminusneuralgie oder andere Formen von Gesichtsschmerzen vorlagen. Der Effekt von Botulinumtoxin A in dieser Studie ist überzeugend. Eine größere Studie ist daher vertretbar, allerdings möglichst mit homogenen Patienten einer Trigeminusneuralgieart und die Verblindung muss sicher gewährleistet sein. Erst dann kann weiter über den Effekt von Botulinumtoxin $A$ bei Trigeminusneuralgie diskutiert werden.

Prof. Dr. med. Stefan Evers

Wu CJ, Lian YJ, Zheng YK, Zhang HF, Chen Y, Xie NC, Wang LJ. Botulinum toxin type A for the treatment of trigeminal neuralgia: results from a randomized, double-blind, placebocontrolled trial. Cephalalgia 2012; 32: 443-50 